\title{
A generalized Alon-Boppana bound and weak Ramanujan graphs
}

\author{
Fan Chung* \\ Department of Mathematics \\ University of California, San Diego \\ La Jolla, CA, U.S.A. \\ fan@ucsd.edu
}

Submitted: Feb 10, 2016; Accepted: Jun 24, 2016; Published: Jul 8, 2016

Mathematics Subject Classifications: 05C50

\begin{abstract}
A basic eigenvalue bound due to Alon and Boppana holds only for regular graphs. In this paper we give a generalized Alon-Boppana bound for eigenvalues of graphs that are not required to be regular. We show that a graph $G$ with diameter $k$ and vertex set $V$, the smallest nontrivial eigenvalue $\lambda_{1}$ of the normalized Laplacian $\mathcal{L}$ satisfies

$$
\lambda_{1} \leqslant 1-\sigma\left(1-\frac{c}{k}\right)
$$

for some constant $c$ where $\sigma=2 \sum_{v} d_{v} \sqrt{d_{v}-1} / \sum_{v} d_{v}^{2}$ and $d_{v}$ denotes the degree of the vertex $v$.

We consider weak Ramanujan graphs defined as graphs satisfying $\lambda_{1} \geqslant 1-\sigma$. We examine the vertex expansion and edge expansion of weak Ramanujan graphs and then use the expansion properties among other methods to derive the above Alon-Boppana bound.
\end{abstract}

\section{Introduction}

The well-known Alon-Boppana bound [8] states that for any $d$-regular graph with diameter $k$, the second largest eigenvalue $\rho$ of the adjacency matrix satisfies

$$
\rho \geqslant 2 \sqrt{d-1}\left(1-\frac{2}{k}\right)-\frac{2}{k}
$$

* Research supported in part by AFSOR FA9550-09-1-0090. 
A natural question is to extend Alon-Boppana bounds for graphs that are irregular. Hoory [6] showed that for an irregular graph, the second largest eigenvalue $\rho$ of the adjacency matrix satisfies

$$
\rho \geqslant 2 \sqrt{d-1}\left(1-\frac{c \log r}{r}\right)
$$

if the average degree of the graph after deleting a ball of radius $r$ is at least $d$ where $r, d>2$.

For irregular graphs, it is often advantageous to consider eigenvalues of the normalized Laplacian for deriving various graph properties. For a graph $G$, the normalized Laplacian $\mathcal{L}$, defined by

$$
\mathcal{L}=I-D^{-1 / 2} A D^{-1 / 2}
$$

where $D$ is the diagonal degree matrix and $A$ denotes the adjacency matrix of $G$. One of the main tools for dealing with general graphs is the Cheeger inequality which relates the least nontrivial eigenvalue $\lambda_{1}$ to the Cheeger constant $h_{G}$ :

$$
2 h_{G} \geqslant \lambda_{1} \geqslant \frac{h_{G}^{2}}{2}
$$

where $h_{G}=\min _{S}|\partial(S)| / \operatorname{vol}(S)$ for $S$ ranging over all vertex subsets with volume $\operatorname{vol}(S)=$ $\sum_{u \in S} d_{u}$ no more than half of $\sum_{u \in V} d_{u}$ and $\partial(S)$ denotes the set of edges leaving $S$. For $k$-regular graphs, we have $\lambda_{1}=1-\rho / k$ where $\rho$ denotes the second largest eigenvalue of the adjacency matrix. In general,

$$
\frac{\rho}{\max _{v} d_{v}} \leqslant 1-\lambda_{1} \leqslant \frac{\rho}{\min _{v} d_{v}}
$$

which can be used to derive a version of the Cheeger inequality involving $\rho$ which is less effective than (2) for irregular graphs.

In this paper, we will show that for a connected graph $G$ with diameter $k, \lambda_{1}$ is upper bounded by

$$
\lambda_{1} \leqslant 1-\sigma\left(1-\frac{c}{k}\right)
$$

for a constant $c$ where $\sigma=2 \sum_{v} d_{v} \sqrt{d_{v}-1} / \sum_{v} d_{v}^{2}$. The above inequality will be proved in Section 6.

The above bound of Alon-Boppana type improves a result of Young [10] who derived a similar eigenvalue bound using a different method. In [10] the notion of $(r, d, \delta)$-robust graphs was considered and it was shown that for a $(r, d, \delta)$-robust graph, the least nontrivial eigenvalue $\lambda_{1}$ satisfies

$$
\lambda_{1} \leqslant 1-\frac{2 d \sqrt{d-1}}{\delta}\left(1-\frac{c}{r}\right) .
$$

Here $(r, d, \delta)$-robustness means for every vertex $v$ and the ball $B_{r}(v)$ consisting of all vertices with distance at most $r$, the induced subgraph on the complement of $B_{r}(v)$ has 
average degree at least $d$ and $\sum_{v \notin B_{r}(v)} d_{v}^{2} /\left|V \backslash B_{r}(v)\right| \leqslant \delta$. We remark that our result in (3) does not require the condition of robustness.

We define weak Ramanujan graphs to be graphs with eigenvalue $\lambda_{1}$ satisfying

$$
\lambda_{1} \geqslant 1-\sigma \geqslant \frac{1}{2}
$$

where $\sigma=2 \sum_{v} d_{v} \sqrt{d_{v}-1} / \sum_{v} d_{v}^{2}$.

To prove the Alon-Boppana bound in (3), it suffices to consider only weak Ramanujan graphs. Weak Ramanujan graphs satisfy various expansion properties. We will describe several vertex-expansion and edge-expansion properties involving $\lambda_{1}$ in Section 3, which will be needed later for proving a diameter bound for weak Ramanujan graphs in Section 4. The diameter bound and related properties of weak Ramanujan graphs are useful in the proof of the Alon-Boppana bound for general graphs.

We will also show that the largest eigenvalue $\lambda_{n-1}$ of the normalized Laplacian satisfies

$$
\lambda_{n-1} \geqslant 1+\sigma\left(1-\frac{c}{k}\right) .
$$

The proof will be given in Section 7 .

\section{Preliminaries}

For a graph $G=(V, E)$, we consider the normalized Laplacian

$$
\mathcal{L}=I-D^{-1 / 2} A D^{-1 / 2}
$$

where $A$ denotes the adjacency matrix and $D$ denotes the diagonal degree matrix with $D(v, v)=d_{v}$, the degree of $v$. We assume that there is no isolated vertex throughout this paper. For a vertex $v$ and a positive integer $l$, let $B_{l}(v)$ denote the ball consisting of all vertices within distance $l$ from $v$. For an edge $\{x, y\} \in E$ we say $x$ is adjacent to $y$ and write $x \sim y$.

Let $\lambda_{0} \leqslant \lambda_{1} \leqslant \ldots \leqslant \lambda_{n-1}$ denote eigenvalues of $\mathcal{L}$, where $n$ denotes the number of vertices in $G$. It can be checked (see [2]) that $\lambda_{1}>0$ if $G$ is connected. The AlonBoppana bound obviously holds if $\lambda_{1}=0$. In the remainder of this paper, we assume $G$ is connected.

Let $\varphi_{i}$ denote the orthonormal eigenvector associated with eigenvalue $\lambda_{i}$. In particular, $\varphi_{0}=D^{1 / 2} \mathbf{1} / \sqrt{\operatorname{vol}(G)}$ where $\mathbf{1}$ is the all 1's vector and $\operatorname{vol}(G)=\sum_{v \in V} d_{v}$. We can then write

$$
\begin{aligned}
\lambda_{1} & =\inf _{g \perp \varphi_{0}} \frac{\langle g, \mathcal{L} g\rangle}{\langle g, g\rangle} \\
& =\inf _{f \perp D \mathbf{1}} \frac{\sum_{x \sim y}(f(x)-f(y))^{2}}{\sum_{z} f^{2}(z) d_{z}} \\
& =\inf _{f \perp D \mathbf{1}} R(f)
\end{aligned}
$$


where $f$ ranges over all functions satisfying $\sum_{u} f(u) d_{u}=0$ and the sum $\sum_{x \sim y}$ ranges over all unordered pairs $\{x, y\}$ where $x$ is adjacent to $y$. Here $R(f)$ denote the Rayleigh quotient of $f$, which can be written as follows:

$$
\begin{aligned}
R(f) & =\frac{\int|\nabla f|}{\int\|f\|^{2}} \\
\text { where } \int\|f\|^{2} & =\sum_{x} f^{2}(x) d_{x} \\
\text { and } \int|\nabla f| & =\sum_{x \sim y}(f(x)-f(y))^{2} .
\end{aligned}
$$

For eigenfunction $\varphi_{i}$, the function $f_{i}=D^{-1 / 2} \varphi_{i}$, called the combinatorial eigenfucntion associated with $\lambda_{i}$, satisfies

$$
\lambda_{i} f(u) d_{u}=\sum_{v \sim u}(f(u)-f(v))
$$

for each vertex $u$. In particular, for $f$ satisfying $\sum_{u} f(u) d_{u}=0$, we have

$$
\langle f, A f\rangle \leqslant\left(1-\lambda_{1}\right)\langle f, D f\rangle
$$

and

$$
|\langle f, A f\rangle| \leqslant \max _{i \neq 0}\left(1-\lambda_{i}\right)\langle f, D f\rangle .
$$

\section{$3 \quad$ Vertex and edge expansions}

For any subset $S$ of vertices, there are two types of boundaries. The edge boundary of $S$, denoted by $\partial(S)$ consists of all edges with exactly one endpoint in $S$. The vertex boundary of $S$, denoted by $\delta(S)$ consists of all vertices not in $S$ but adjacent to vertices in $S$. Namely,

$$
\begin{aligned}
& \partial(S)=\{\{u, v\} \in E: u \in S \text { and } v \notin S\}=E(S, \bar{S}) \\
& \delta(S)=\{u \notin S: u \sim v \in S \text { for some vertex } v\}
\end{aligned}
$$

In this section, we will examine vertex expansion and edge expansion relying only on $\lambda_{1}$. These expansion properties will be needed for deriving diameter bounds for weak Ramanujan graphs which will be used in our proof of the general Alon-Boppana bound later in Section 6.

From the definition of the Cheeger constant, for all vertex subsets $S$, we have

$$
\frac{|\partial(S)|}{\operatorname{vol}(S)} \geqslant h_{G} \geqslant \frac{\lambda_{1}}{2}
$$

Later in the proofs, we will be interested in the case that $\operatorname{vol}(S)$ is small and therefore we will use the following version. 
Lemma 1. Let $S$ be a subset of vertices in $G$. Then

$$
\frac{|\partial(S)|}{\operatorname{vol}(S)} \geqslant \lambda_{1}\left(1-\frac{\operatorname{vol}(S)}{\operatorname{vol}(G)}\right)
$$

Proof. Suppose $f$ is defined by

$$
f=\frac{\mathbf{1}_{S}}{\operatorname{vol}(S)}-\frac{\mathbf{1}_{\bar{S}}}{\operatorname{vol}(\bar{S})}
$$

where $\mathbf{1}_{S}$ denotes the characteristic function defined by $\mathbf{1}_{S}(v)=1$ if $v \in S$ and 0 otherwise.

The Rayleigh quotient $R(f)$ satisfies

$$
\lambda_{1} \leqslant R(f)=\frac{|\partial(S)|}{\operatorname{vol}(S)} \cdot \frac{\operatorname{vol}(G)}{\operatorname{vol}(\bar{S})} .
$$

For the expansion of the vertex boundary, the Tanner bound [9] for regular graphs can be generalized as follows.

Lemma 2. Let $\bar{\lambda}=\min _{i \neq 0}\left|1-\lambda_{i}\right|$. Then for any vertex subset $S$ in a graph,

$$
\frac{\operatorname{vol}(\delta(S))}{\operatorname{vol}(S)} \geqslant \frac{1-\bar{\lambda}^{2}}{\bar{\lambda}^{2}+\frac{\operatorname{vol}(S)}{\operatorname{vol}(\bar{S})}}
$$

The proof of the above inequality is by using the following discrepancy inequality (as seen in $[2])$.

Lemma 3. In a graph $G$, for two subset $X$ and $Y$ of vertices, the number $e(X, Y)=$ $|E(X, Y)|$ of edges between $X$ and $Y$ satisfies

$$
\left|e(X, Y)-\frac{\operatorname{vol}(X) \operatorname{vol}(Y)}{\operatorname{vol}(G)}\right| \leqslant \bar{\lambda} \frac{\sqrt{\operatorname{vol}(X) \operatorname{vol}(Y) \operatorname{vol}(\bar{X}) \operatorname{vol}(\bar{Y})}}{\operatorname{vol}(G)}
$$

where $\bar{\lambda}=\min _{i \neq 0}\left|1-\lambda_{i}\right|$.

The proof of Lemma 3 follows from (9) and can be found in [2]. The proof of (12) results from (11) by setting $X=S$ and $Y=\overline{S \cup \delta(S)}$.

Here we will give a version of the vertex-expansion bounds for general graphs which only rely on $\lambda_{1}$ and are independent of other eigenvalues.

Lemma 4. In a graph $G$ with vertex set $V$ and the first nontrivial eigenvalue $\lambda_{1}$, for a subset $S$ of $V$ with $\operatorname{vol}(S \cup \delta S) \leqslant \epsilon \operatorname{vol}(G) \leqslant \operatorname{vol}(G) / 2$, the vertex boundary of $S$ satisfies

$$
\frac{\operatorname{vol}(\delta(S))}{\operatorname{vol}(S)} \geqslant \frac{2 \lambda_{1}}{1-\lambda_{1}+2 \epsilon}
$$

(ii) If $1 / 2 \leqslant \lambda_{1} \leqslant 1-2 \epsilon$, then

$$
\frac{\operatorname{vol}(\delta(S))}{\operatorname{vol}(S)} \geqslant \frac{1}{\left(1-\lambda_{1}+2 \epsilon\right)^{2}}
$$


Proof. The proof of (i) follows from Lemma 1 since

$$
\begin{aligned}
\frac{\operatorname{vol}(\delta(S))}{\operatorname{vol}(S)} & \geqslant \frac{|\partial(S \cup \delta(S))|+|\partial(S)|}{\operatorname{vol}(S)} \\
& \geqslant \frac{\lambda_{1}(1-\epsilon)\left(\operatorname{vol}(S)+\operatorname{vol}(\delta(S))+\lambda_{1}(1-\epsilon) \operatorname{vol}(S)\right.}{\operatorname{vol}(S)}
\end{aligned}
$$

Therefore

$$
\frac{\operatorname{vol}(\delta(S))}{\operatorname{vol}(S)} \geqslant \frac{2 \lambda_{1}(1-\epsilon)}{1-\lambda_{1}(1-\epsilon)} \geqslant \frac{2 \lambda_{1}}{1-\lambda_{1}+2 \epsilon}
$$

To prove (ii), we set $f=\mathbf{1}_{S}+\gamma \mathbf{1}_{\delta(S)}$ where $\gamma=1-\lambda_{1}$. Consider $g=f-c \mathbf{1}_{V}$ where $c=\sum_{u} f(u) d_{u} / \operatorname{vol}(G)$. By the Cauchy-Schwarz inequality, we have

$$
\begin{aligned}
c^{2}=\frac{1}{(\operatorname{vol}(G))^{2}}\left(\sum_{u \in S \cup \delta(S)} f(u) d_{u}\right)^{2} & \leqslant \frac{\operatorname{vol}(S \cup \delta(S))}{(\operatorname{vol}(G))^{2}} \sum_{u} f^{2}(u) d_{u} \\
& \leqslant \frac{\epsilon}{\operatorname{vol}(G)} \sum_{u} f^{2}(u) d_{u} .
\end{aligned}
$$

Using the inequality in (8), we have

$$
\begin{aligned}
\langle f, A f\rangle & \leqslant\langle g, A g\rangle+c^{2} \operatorname{vol}(G) \\
& \leqslant \gamma\langle g, D g\rangle+c^{2} \operatorname{vol}(G) \\
& =\gamma\langle f, D f\rangle+(1-\gamma) c^{2} \operatorname{vol}(G) \\
& \leqslant(\gamma+\epsilon)\langle f, D f\rangle \\
& =(\gamma+\epsilon)\left(\operatorname{vol}(S)+\gamma^{2} \operatorname{vol}(\delta(S))\right) .
\end{aligned}
$$

Let $e(S, T)$ denote the number of ordered pairs $(u, v)$ where $u \in S, v \in T$ and $\{u, v\} \in E$. Since $\gamma=1-\lambda \leqslant 1 / 2$, we have

$$
\begin{aligned}
\langle f, A f\rangle & \geqslant e(S, S)+2 \gamma e(S, \delta(S)) \\
& \geqslant(1-2 \gamma) e(S, S)+2 \gamma \operatorname{vol}(S) \\
& \geqslant 2 \gamma \operatorname{vol}(S)
\end{aligned}
$$

Together we have

$$
\begin{aligned}
\frac{\operatorname{vol}(\delta(S))}{\operatorname{vol}(S)} & \geqslant \frac{\gamma-\epsilon}{\sigma^{2}(\gamma+\epsilon)} \\
& \geqslant \frac{1}{(\gamma+2 \epsilon)^{2}}
\end{aligned}
$$

since $\gamma \geqslant 2 \epsilon$. 
Recall that weak Ramanujan graphs have eigenvalue $\lambda_{1}$ satisfying

$$
\lambda_{1} \geqslant 1-\sigma
$$

where $\sigma=2 \sum_{v} d_{v} \sqrt{d_{v}-1} / \sum_{v} d_{v}^{2}$. Lemma 1 implies that for $S$ with $\operatorname{vol}(S \cup \delta(S)) \leqslant$ $\epsilon \operatorname{vol}(G)$,

$$
\frac{\operatorname{vol}(\delta(S))}{\operatorname{vol}(S)} \geqslant \frac{1}{(\sigma+2 \epsilon)^{2}}
$$

For $k$-regular Ramanujan graphs with eigenvalue $\lambda_{1}=1-2 \sqrt{k-1} / k$, the above inequality is consistent with the bound

$$
\frac{\operatorname{vol}(\delta(S))}{\operatorname{vol}(S)}=\frac{|\delta(S)|}{|S|} \geqslant \frac{1}{\left(\frac{2 \sqrt{k-1}}{k}+2 \epsilon\right)^{2}}
$$

which is about $k / 4$ when $\operatorname{vol}(S)$ is small. The factor $k / 4$ in the above inequality was improved by Kahale [4] to $k / 2$. There are many applications (see [1]) that require graphs having expansion factor to be $(1-\epsilon) k$. Such graphs are called lossless expanders. In [1], lossless graphs were constructed explicitly by using the zig-zag construction but the method for deriving the expansion bounds does not use eigenvalues. In this paper, the expansion factor as in Lemma 4 is enough for our proof later.

\section{Weak Ramanujan graphs}

We recall that a graph is said to be a weak Ramanujan graph as in (14) if

$$
\lambda_{1} \geqslant 1-\sigma \geqslant \frac{1}{2}
$$

where

$$
\sigma=2 \frac{\sum_{v} d_{v} \sqrt{d_{v}-1}}{\sum_{v} d_{v}^{2}}
$$

To prove the Alon-Boppana bound, it is enough to consider only weak Ramanujan graphs.

Lemma 5. As defined in (15), $\sigma$ satisfies

$$
\frac{2 \sqrt{\bar{d}-1}}{\breve{d}} \leqslant \sigma \leqslant \frac{2 \sqrt{\bar{d}-1}}{\bar{d}}
$$

where $\bar{d}$ denotes the average degree in $G$ and $\breve{d}$ denote the second order degree, i.e.,

$$
\bar{d}=\frac{\sum_{v} d_{v}}{n} \quad \text { and } \quad \breve{d}=\frac{\sum_{v} d_{v}^{2}}{\sum_{v} d_{v}} .
$$


Proof. The proof is mainly by using the Cauchy-Schwarz inequality. For the upper bound, we note that

$$
\begin{aligned}
\sigma=2 \frac{\sum_{v} d_{v} \sqrt{d_{v}-1}}{\sum_{v} d_{v}^{2}} & \leqslant 2 \frac{\sqrt{\sum_{v} d^{2} \sum_{v}\left(d_{v}-1\right)}}{\sum_{v} d_{v}^{2}} \\
& =2 \frac{\sqrt{\sum_{v}\left(d_{v}-1\right)}}{\sqrt{\sum_{v} d_{v}^{2}}} \\
& \leqslant 2 \frac{\sqrt{\sum_{v}\left(d_{v}-1\right)}}{\sum_{v} d_{v} / \sqrt{n}} \\
& \leqslant 2 \frac{\sqrt{\sum_{v}\left(d_{v}-1\right)}}{\bar{d} \sqrt{n}} \leqslant \frac{2 \sqrt{\bar{d}-1}}{\bar{d}} .
\end{aligned}
$$

For the upper bound, we will use the fact that for $a, b>1$ and $a+b=c$,

$$
a \sqrt{a-1}+b \sqrt{b-1} \geqslant c \sqrt{\frac{c}{2}-1}
$$

and therefore

$$
\sum_{v} d_{v} \sqrt{d_{v}-1} \geqslant \sum_{v} d_{v} \sqrt{\frac{\sum_{v} d_{v}}{n}-1}
$$

Consequently, we have

$$
\sigma=2 \frac{\sum_{v} d_{v} \sqrt{d_{v}-1}}{\sum_{v} d_{v}^{2}} \geqslant 2 \frac{\sum_{v} d_{v} \sqrt{\frac{\sum_{v} d_{v}}{n}-1}}{\frac{\sum_{v} d_{v}^{2}}{\sum_{v} d_{v}} \sum_{v} d_{v}} \geqslant 2 \frac{\sqrt{\bar{d}-1}}{\breve{d}}
$$

as desired.

We remark that for graphs with average degree at least 20, we have $\sigma<1 / 2<\lambda_{1}$.

Theorem 6. Suppose a weak Ramanujan graph $G$ has diameter $k$. Then for any $\epsilon>0$, we have

$$
k \leqslant(1+\epsilon) \frac{2 \log \operatorname{vol}(G)}{\log \sigma^{-1}}
$$

provided that the volume of $G$ is large, i.e., $\operatorname{vol}(G) \geqslant c \sigma^{\log (\sigma)} / \epsilon$ for some small constant $c$. Proof. We set

$$
t=\left\lceil(1+\epsilon) \frac{\log (\operatorname{vol}(G))}{\log \sigma^{-1}}\right\rceil .
$$

It suffices to show that for every vertex $v$, the ball $B_{t}(v)$ has volume more than $\operatorname{vol}(G) / 2$.

Suppose $\operatorname{vol}\left(B_{t}(v)\right) \leqslant \operatorname{vol}(G) / 2$. Let

$$
s_{j}=\frac{\operatorname{vol}\left(B_{j}(u)\right)}{\operatorname{vol}(G)} .
$$


By part (i) of Lemma 4, we have $\operatorname{vol}\left(\delta\left(B_{u}(j)\right)\right) \geqslant 0.5 \operatorname{vol}\left(B_{u}(j)\right)$ for $j \leqslant t-1$ and therefore $s_{j+1} \geqslant 1.5 s_{j}$. Thus, if $j \leqslant t-c_{1} \log \left(\sigma^{-1}\right)$, then $s_{j} \leqslant \sigma^{4}$ where $c_{1}$ is some small constant satisfying $c_{1} \leqslant 4(\log 1.5)^{-1}$.

Now we apply part (ii) of Lemma 4 and we have, for $j \leqslant t-c_{1} \log \left(\sigma^{-1}\right)$,

$$
\frac{s_{j+1}}{s_{j}}=\frac{\operatorname{vol}\left(B_{j+1}(u)\right)}{\operatorname{vol}\left(B_{j}(u)\right)} \geqslant \frac{\operatorname{vol}\left(\delta\left(B_{j}(u)\right)\right)}{\operatorname{vol}\left(B_{j}(u)\right)} \geqslant \frac{1}{\left(\sigma+2 s_{j}\right)^{2}} \geqslant \frac{1}{\left(\sigma+2 \sigma^{4}\right)} .
$$

This implies, for $l \leqslant t-c_{1} \log \left(\sigma^{-1}\right)$,

$$
\begin{aligned}
\frac{s_{l}}{s_{0}} \geqslant \prod_{0<j<l} \frac{1}{\left(\sigma+2 s_{j}\right)^{2}} & \geqslant \prod_{0<j<l} \frac{1}{\left(\sigma+2 \sigma^{4}\right)^{2}} \\
& \geqslant \frac{1}{\sigma^{2 l}\left(1+2 \sigma^{4}\right)^{2 l}} .
\end{aligned}
$$

Since $s_{0} \geqslant 1 / \operatorname{vol}(G)$ and $s_{l} \leqslant s_{t} \leqslant 1 / 2$, we have

$$
\operatorname{vol}(G) \geqslant \frac{1}{\sigma^{2 l}\left(1+2 \sigma^{4}\right)^{2 l}}
$$

Hence

$$
l \leqslant \frac{\log (\operatorname{vol}(G))}{\log \left(\sigma^{-1}\right)+2 \sigma^{4}}
$$

However,

$$
(1+\epsilon) \frac{\log (\operatorname{vol}(G))}{\log \left(\sigma^{-1}\right)} \leqslant t \leqslant c_{1} \log \left(\sigma^{-1}\right)+\frac{\log (\operatorname{vol}(G))}{\log \left(\sigma^{-1}\right)+2 \sigma^{4}}
$$

which is a contracdiction for $G$ with $\operatorname{vol}(G)$ large, say, $\operatorname{vol}(G) \geqslant \sigma^{2 c_{1} \log \sigma} / \epsilon$. Thus we conclude that $s_{t} \geqslant 1 / 2$ and Theorem 6 is proved.

Theorem 7. For a weak Ramanujan graph with diameter $k$, for any vertex $v$ and any $l \leqslant k / 4$, the ball $B_{u}(l)$ has volume at most $\epsilon \operatorname{vol}(G)$ if $k \geqslant c \log \epsilon^{-1}$, for some constants $c$.

Proof. We will prove by contradiction. Suppose that for $j_{0}=\lceil k / 4\rceil$, there is a vertex $u$ with $\operatorname{vol}\left(B_{v}\left(j_{0}\right)\right)>\epsilon \operatorname{vol}(G)$. Let $r$ denote the largest integer such that

$$
s_{r}=\frac{\operatorname{vol}\left(B_{u}(r)\right)}{\operatorname{vol}(G)}>\frac{1}{2} .
$$

By the assumption, we have $r>k / 4$ and $s_{j_{0}}>\epsilon$. There are two possibilities:

Case 1: $r \geqslant k / 2$.

By part (i) of Lemma 4, we have $\operatorname{vol}\left(\delta\left(B_{u}(j)\right)\right) \geqslant 0.5 \operatorname{vol}\left(B_{u}(j)\right)$ for $j \leqslant k / 2$ and therefore $s_{j+1} \geqslant 1.5 s_{j}$. Thus, for $j \leqslant k / 2-c_{1} \log \epsilon^{-1}$, we have $s_{j} \leqslant \epsilon$ where $c_{1}=1 / \log 1.5$. Since $k / 4 \leqslant k / 2-c_{1} \log \epsilon^{-1}$, we have a contradiction. 
Case 2: $r<k / 2$.

We define

$$
\bar{s}_{j}=\frac{\operatorname{vol}\left(V \backslash B_{u}(j)\right)}{\operatorname{vol}(G)} .
$$

Thus $\bar{s}_{j}<1 / 2$ for all $j \geqslant k / 2$. We consider two subcases.

Subcase 2a: Suppose $\bar{s}_{j} \geqslant \epsilon$ for $j \geqslant k / 2$.

Using Lemma 4 , for $j$ where $r \leqslant j \leqslant k / 2$, we have $\bar{s}_{j} \geqslant 1.5 \bar{s}_{j+1}$. Thus, for some $j_{1} \geqslant k / 2-c_{1} \log \epsilon^{-1}$, we have $\bar{s}_{j} \geqslant 1 / 2$ or equivalently, $s_{j} \leqslant 1 / 2$. By using Lemma 4 again, for $j \leqslant j_{1}$, we have $s_{j+1} \geqslant 1.5 s_{j}$ and therefore for any $j \leqslant j_{1}-c_{1} \log \epsilon^{-1}$ we have $s_{j} \leqslant \epsilon$. Since $j_{1}-c_{1} \log \epsilon^{-1} \geqslant k / 2-2 c_{1} \log \epsilon^{-1} \geqslant k / 4$, we again have a contradiction to the assumption $s_{j_{0}} \geqslant \epsilon$.

Subcase 2b: Suppose $\bar{s}_{j}<\epsilon$ for $j \geqslant k / 2$

We apply part (ii) of Lemma 4 and we have, for $j \geqslant k / 2$,

$$
\frac{\bar{s}_{j}}{\bar{s}_{j+1}} \geqslant \frac{1}{(\sigma+2 \epsilon)^{2}} \text {. }
$$

This implies, for $j_{2}=\lceil k / 2\rceil$,

$$
\frac{\bar{s}_{j_{2}}}{\bar{s}_{k}} \geqslant \prod_{k / 2<j \leqslant k} \frac{1}{\left(\sigma+2 s_{j}\right)^{2}} \geqslant \frac{1}{(\sigma+2 \epsilon)^{k}} .
$$

Since $\bar{s}_{k} \geqslant 1 / \operatorname{vol}(G)$, we have

$$
\bar{s}_{j_{1}} \geqslant \frac{1}{\operatorname{vol}(G)(\sigma+2 \epsilon)^{k}} .
$$

Since the assumption of this subcase is $\bar{s}_{j_{1}}<\epsilon$, we have

$$
k \geqslant \frac{\log n+\log \epsilon^{-1}}{\log \sigma^{-1}} .
$$

We now use Lemma 4 and we have, for $j=k / 2-j^{\prime} \geqslant r$

$$
\bar{s}_{j} \geqslant \frac{1}{\operatorname{vol}(G)(\sigma+2 \epsilon)^{k+2 j^{\prime}}} .
$$

Therefore, for some $j \leqslant k / 2-\log \epsilon^{-1} / \log \sigma^{-1}$, we have $\bar{s}_{j}>1 / 2$ which implies $r \geqslant$ $k / 2-\log \epsilon^{-1} / \log \sigma^{-1}$.

Now we use the same argument as in Case 1 except shifting $r$ by $\log \epsilon^{-1} / \log \sigma^{-1}$. For some $j \leqslant r-c_{1} \log \epsilon^{-1} \leqslant k / 2-\log \epsilon^{-1} / \log \sigma^{-1}-c_{1} \log \epsilon^{-1}$, we have $s_{j}<\epsilon$. Since $\log \epsilon^{-1} / \log \sigma^{-1}+c_{1} \log \epsilon^{-1}<k / 4$, this leads to a contradiction and Theorem 7 is proved. 


\section{Non-backtracking random walks}

Before we proceed to the proof of the Alon-Boppana bound, we will need some basic facts on non-backtracking random walks.

A non-backtracking walk is a sequence of vertices $\mathbf{p}=\left(v_{0}, v_{1}, \ldots, v_{t}\right)$ for some $t$ such that $v_{i-1} \sim v_{i}$ and $v_{i+1} \neq v_{i-1}$ for $i=1, \ldots, t-2$. The non-backtracking random walk can be described as follows: For $i \geqslant 1$, at the $i$ th step on $v_{i}$, choose with equal probability a neighbor $u$ of $v_{i}$ where $u \neq v_{i-1}$, move to $u$ and set $v_{i+1}=u$. To simplify notation, we call a non-backtracking walk an NB-walk. The modified transition probability matrix $\tilde{P}_{k}$, for $k=0,1, \ldots, t-1$, is defined by

$$
\tilde{P}_{k}(u, v)= \begin{cases}P^{k}(u, v) & \text { if } k=0 \\ \sum_{\mathbf{p} \in \mathscr{P}_{u, v}^{(k)}} w(\mathbf{p}) & \text { if } k \geqslant 1\end{cases}
$$

where the weight $w(\mathbf{p})$ for an NB-walk $\mathbf{p}=\left(v_{0}, v_{1}, \ldots, v_{t}\right)$ with $t \geqslant 1$ is defined to be

$$
w(\mathbf{p})=\frac{1}{d_{v_{0}} \prod_{i=1}^{t-1}\left(d_{v_{i}}-1\right)}
$$

and $\mathscr{P}_{u, v}^{(k)}$ denotes the set of non-backtracking walks from $u$ to $v$. For a walk $\mathbf{p}=\left(v_{0}\right)$ of length 0 , we define $w(\mathbf{p})=1$.

Although a non-backtracking random walk is not a Markov chain, it is closely related to an associated Markov chain as we will describe below (also see [6]).

For each edge $\{u, v\}$ in $E$, we consider two directed edges $(u, v)$ and $(v, u)$. Let $\hat{E}$ denote the set consisting of all such directed edges, i.e. $\hat{E}=\{(u, v):\{u, v\} \in E\}$. We consider a random walk on $\hat{E}$ with transition probability matrix $\boldsymbol{P}$ defined as follows:

$$
\boldsymbol{P}\left((u, v),\left(u^{\prime}, v^{\prime}\right)\right)= \begin{cases}\frac{1}{d_{v}-1} & \text { if } v=u^{\prime} \text { and } u \neq v^{\prime} \\ 0 & \text { otherwise. }\end{cases}
$$

Let $\mathbf{1}_{E}$ denote the all 1's function defined on the edge set $E$ as a row vector. From the above definition, we have

$$
\mathbf{1}_{E} \boldsymbol{P}=\mathbf{1}_{E}
$$

In addition, we define the vertex-edge incidence matrix $B$ and $B^{*}$ for $a \in V$ and $(b, c) \in \hat{E}$ by

$$
\begin{gathered}
B(a,(b, c))= \begin{cases}1 & \text { if } a=b, \\
0 & \text { otherwise }\end{cases} \\
\left.B^{*}((b, c), a)\right)= \begin{cases}1 & \text { if } c=a, \\
0 & \text { otherwise. }\end{cases}
\end{gathered}
$$


Let $\mathbf{1}_{V}$ denote all 1's vector defined on the vertex set $V$. Then

$$
\mathbf{1}_{V} B=\mathbf{1}_{E}
$$

Although $\tilde{P}_{k}$ is not a Markov chain, it is related to the Markov chain determined by $\boldsymbol{P}$ on $\hat{E}$ as follows:

Fact 1: For $l \geqslant 1$.

$$
\tilde{P}_{l}=D^{-1} B \boldsymbol{P}^{l} B^{*}
$$

and for the case of $l=0$, we have $\tilde{P}_{0}=I$.

By combining (19) and (20), we have

Fact 2:

$$
\mathbf{1}_{V} D \tilde{P}_{l}=\mathbf{1}_{E} B^{*}=\mathbf{1}_{V} D .
$$

Note that $\mathbf{1}_{V} D$ is just the degree vector for the graph $G$. Therefore (21) states that the degree vector is an eigenvector of $\tilde{P}_{l}$. Using Fact 1 and 2, we have the following:

\section{Lemma 8.}

(i) For a fixed vertex $x$ and any integer $j \geqslant 0$, we have

$$
\sum_{u} d_{u} \sum_{\mathbf{p} \in \mathscr{P} u, x}^{(j)} w(\mathbf{p})=d_{x}
$$

(ii) For a fixed vertex $u$, we have

$$
\sum_{x} \sum_{\mathbf{p} \in \mathscr{P}_{u, x}^{(j)}} w(\mathbf{p})=\mathbf{1}_{u}\left(I+\tilde{P}_{1}+\ldots+\tilde{P}_{l}\right) \mathbf{1}^{*}=l+1
$$

where $\mathbf{1}_{u}$ denotes the characteristic function which assumes value 1 at $u$ and 0 else where. Proof. The proof of (22) and (23) follows from the fact that

$$
\mathbf{1}_{V} D \tilde{P}_{j}(x)=\mathbf{1}_{V} D\left(D^{-1} B \mathbf{P}^{j} B^{*}\right)=\mathbf{1}_{E} \mathbf{P}^{j} B^{*}=\mathbf{1}_{E} B^{*}=\mathbf{1}_{V} D(x)
$$

and $\mathbf{1}_{u} \tilde{P}_{j}(x)=w(\mathbf{p})$ for $\mathbf{p} \in \mathscr{P}_{u, x}^{(j)}$.

\section{An Alon-Boppana bound for $\lambda_{1}$}

Theorem 9. In a graph $G=(V, E)$ with diameter $k$, the first nontrivial eigenvalue $\lambda_{1}$ satisfies

$$
\lambda_{1} \leqslant 1-\sigma\left(1-\frac{c}{k}\right)
$$

where $\sigma$ is as defined in (15), provided $k \geqslant c^{\prime} \log \sigma^{-1}$ and $\operatorname{vol}(G) \geqslant c^{\prime \prime} \sigma^{\log \sigma}$ for some absolute constants c's. 
Proof. If $G$ is not a weak Ramanujan graph, we have $\lambda_{1} \leqslant 1-\sigma$ and we are done. We may assume that $G$ is weak Ramanujan.

From the definition of $\lambda_{1}$, we have

$$
\lambda_{1} \leqslant \frac{\sum_{x \sim y}(f(x)-f(y))^{2}}{\sum_{x} f^{2}(x) d_{x}}=R(f)
$$

where $f$ satisfies $\sum_{x} f(x) d_{x}=0$.

We will construct an appropriate $f$ satisfying $R(f) \leqslant 1-\sigma(1-c / k)$ and therefore serve as an upper bound for $\lambda_{1}$. We set

$$
t=\left\lfloor\frac{\log (\operatorname{vol}(G))}{\log \sigma^{-1}}\right\rfloor
$$

and choose $\epsilon$ satisfying

$$
\epsilon \leqslant \frac{\sigma}{t} \leqslant \frac{c \sigma}{k}
$$

by using Theorem 6 where $\sigma$ is as defined in (15).

We consider a family of functions defined as follows. For a specified vertex $u$ and an integer $l=\lfloor k / 4\rfloor$, we consider a function $g_{u}: V \rightarrow \mathbb{R}^{+}$, defined by

$$
\begin{aligned}
g_{u}(x) & =\left(\mathbf{1}_{u}\left(I+\tilde{P}_{1}+\ldots+\tilde{P}_{l}\right)(x)\right)^{1 / 2} \\
& =\left(\sum_{j=0}^{l} \sum_{\mathbf{p} \in \mathscr{P}_{u, x}^{(j)}} w(\mathbf{p})\right)^{1 / 2}
\end{aligned}
$$

where $\tilde{P}_{j}$ is as defined in (20) and $\mathbf{1}_{u}$ is treated as a row vector. In other words, $g_{u}$ denotes the square root of the sum of non-backtracking random walks starting from $u$ taking $i$ steps for $i$ ranging from 0 to $l$.

Claim A:

$$
\sum_{u} d_{u} \sum_{x} g_{u}^{2}(x) d_{x}=\sum_{j=0}^{l} \sum_{x} \sum_{\mathbf{p} \in \mathscr{P}_{u, x}^{(l)}} d_{u} w(\mathbf{p}) d_{x}=(l+1) \sum_{x} d_{x}^{2}
$$

where the weight $w(\mathbf{p})$ of a walk $\mathbf{p}$ is as defined in (17).

Proof of Claim A: From the definition of $g_{u}$ and (16), we have

$$
\begin{aligned}
\sum_{u} d_{u} \sum_{x} g_{u}^{2}(x) d_{x} & =\sum_{j=0}^{l} \sum_{x} \sum_{\mathbf{p} \in \mathscr{P}_{u, x}^{(l)}} d_{u} w(\mathbf{p}) \\
& =\sum_{u} d_{u} \mathbf{1}_{u} B\left(I+\tilde{P}_{1}+\ldots+\tilde{P}_{l}\right)(x)
\end{aligned}
$$




$$
\begin{aligned}
& =\sum_{u} d_{u} \sum_{i=1}^{l} \mathbf{1}_{u} D^{-1} B \mathbf{P}^{i} B^{*}(x) d_{x}+\sum_{x} d_{x}^{2} \\
& =\sum_{i=1}^{l} \sum_{u} \mathbf{1}_{u} B \mathbf{P}^{i} B^{*}(x) d_{x}+\sum_{x} d_{x}^{2} \\
& =\sum_{i=1}^{l} \mathbf{1}_{E} \mathbf{P}^{i} B^{*}(x) d_{x}+\sum_{x} d_{x}^{2} \\
& =l \mathbf{1}_{E} B^{*}(x) d_{x}+\sum_{x} d_{x}^{2} \\
& =(l+1) \sum_{x} d_{x}^{2} .
\end{aligned}
$$

Claim A is proved.

Claim B:

$$
\sum_{u} d_{u} \sum_{x \sim y}\left(g_{u}(x)-g_{u}(y)\right)^{2} \leqslant(l+1-l \sigma) \sum_{x} d_{x}^{2}
$$

where $\sum_{x \sim y}$ denotes the sum ranging over unordered pairs $\{x, y\}$ where $x$ is adjacent to $y$.

\section{Proof of Claim B:}

We will use the following fact for $a_{i}, b_{i}>0$.

$$
\left(\sqrt{\sum_{i} a_{i}}-\sqrt{\sum_{i} b_{i}}\right)^{2} \leqslant \sum_{i}\left(\sqrt{a_{i}}-\sqrt{b_{i}}\right)^{2}
$$

which can be easily checked.

For a fixed vertex $u$, we apply Claim B:

$$
\begin{aligned}
& \sum_{x \sim y}\left(g_{u}(x)-g_{u}(y)\right)^{2} \\
& =\sum_{x \sim y}\left(\sqrt{\left.\sum_{\substack{\mathbf{p} \in \mathscr{P}_{u, x}^{(t)} \\
t \leqslant l}}^{(t)}-\sqrt{\sum_{\substack{\mathbf{p}^{\prime} \in \mathscr{P}_{u}^{(t)} \\
t \leqslant l}}^{(t)} w\left(\mathbf{p}^{\prime}\right)}\right)^{2}}\right. \\
& \leqslant \sum_{t \leqslant l-1} \sum_{r \in V} \sum_{\substack{\mathbf{p} \in \mathscr{P}_{u, r}^{(t)} \\
\mathbf{p}^{\prime}=\mathbf{p} \cup s \in \mathscr{P}_{u, s}^{(t+1)}}}\left(\sqrt{w(\mathbf{p})}-\sqrt{w\left(\mathbf{p}^{\prime}\right)}\right)^{2}+\sum_{\mathbf{p} \in \mathscr{P}_{u, x}^{(l)}} w(\mathbf{p})\left(d_{x}-1\right) \\
& \leqslant \sum_{t \leqslant l-1} \sum_{x} \sum_{\mathbf{p} \in \mathscr{P}_{u, x}^{(t)}}\left(\sqrt{w(\mathbf{p})}-\sqrt{\frac{w(\mathbf{p})}{d_{x}-1}}\right)^{2}\left(d_{x}-1\right)+\sum_{\mathbf{p} \in \mathscr{P}_{u, x}^{(l)}} \sqrt{w(\mathbf{p})}\left(d_{x}-1\right)
\end{aligned}
$$




$$
\begin{aligned}
& \leqslant \sum_{t \leqslant l-1} \sum_{x} \sum_{\mathbf{p} \in \mathscr{P}_{u, x}^{(t)}} w(\mathbf{p})\left(1+\frac{1}{d_{x}-1}-\frac{2}{\sqrt{d_{x}-1}}\right)\left(d_{x}-1\right)+\sum_{\mathbf{p} \in \mathscr{P}_{u, x}^{(l)}} w(\mathbf{p})\left(d_{x}-1\right) \\
& \leqslant \sum_{t \leqslant l-1} \sum_{x} \sum_{\mathbf{p} \in \mathscr{P}_{u, x}^{(t)}} w(\mathbf{p})\left(d_{x}-2 \sqrt{d_{x}-1}\right)+\sum_{\mathbf{p} \in \mathscr{P}_{u, x}^{(l)}} w(\mathbf{p})\left(d_{x}-1\right) .
\end{aligned}
$$

Using Fact 3, we have

$$
\begin{aligned}
& \sum_{u} d_{u} \sum_{x \sim y}\left(g_{u}(x)-g_{u}(y)\right)^{2} \\
& \leqslant \sum_{t \leqslant l-1} \sum_{u} d_{u} \sum_{\mathbf{p} \in \mathscr{P}_{u, x}^{(t)}} w(\mathbf{p})\left(d_{x}-2 \sqrt{d_{x}-1}\right)+\sum_{u} d_{u} \sum_{\mathbf{p} \in \mathscr{P}_{u, x}^{(l)}} w(\mathbf{p})\left(d_{x}-1\right) \\
& =l \sum_{x} d_{x}\left(d_{x}-2 \sqrt{d_{x}-1}\right)+\sum_{x} d_{x}^{2} \\
& =l(1-\sigma) \sum_{x} d_{x}^{2}+\sum_{x} d_{x}^{2} \\
& =(l+1-l \sigma) \sum_{x} d_{x}^{2}
\end{aligned}
$$

This proves Claim B.

Claim $C$ : There is a vertex $u$ satisfying

$$
R\left(g_{u}\right) \leqslant 1-\sigma\left(1-\frac{1}{l+1}\right)
$$

Proof of Claim C:

Combining Claim A and B, we have

$$
\begin{aligned}
& \sum_{u} d_{u} \sum_{x \sim y}\left(g_{u}(x)-g_{u}(y)\right)^{2} \\
& \leqslant(l+1-l \sigma) \sum_{x} d_{x}^{2} \\
& \leqslant(l+1-l \sigma)\left(\frac{1}{l+1}\right) \sum_{u} d_{u} \sum_{x} g_{u}^{2}(x) d_{x} \\
& =\left(1-\frac{l \sigma}{l+1}\right) \sum_{u} d_{u} \sum_{x} g_{u}^{2}(x) d_{x}
\end{aligned}
$$

Thus we deduce that there is a vertex $u$ such that

$$
\begin{aligned}
R\left(g_{u}\right) & =\frac{\sum_{x \sim y}\left(g_{u}(x)-g_{u}(y)\right)^{2}}{\sum_{x} g_{u}^{2}(x) d_{x}} \\
& \leqslant 1-\frac{l \sigma}{l+1} .
\end{aligned}
$$


We define

$$
\alpha_{v}=\frac{\sum_{x} g_{v}(x) d_{x}}{\sum_{x} d_{x}}=\frac{\sum_{x} g_{v}(x) d_{x}}{\operatorname{vol}(G)}
$$

We consider the function $g_{u}^{\prime}$ defined by

$$
g_{u}^{\prime}(x)=g_{u}(x)-\alpha_{u}
$$

Clearly, $g_{u}^{\prime}$ satisfies the condition that

$$
\sum_{x} g_{u}^{\prime}(x) d_{x}=0
$$

Hence, we have

$$
\begin{aligned}
\lambda_{1} \leqslant R\left(g_{u}^{\prime}\right) & =\frac{\sum_{x \sim y}\left(g_{u}^{\prime}(x)-g_{u}^{\prime}(y)\right)^{2}}{\sum_{x} g_{u}^{\prime 2}(x) d_{x}} \\
& =\frac{\sum_{x \sim y}\left(g_{u}(x)-g_{u}(y)\right)^{2}}{\sum_{x} g_{u}^{2}(x) d_{x}-\alpha_{u}^{2} \operatorname{vol}(G)} .
\end{aligned}
$$

Note that by the Cauchy-Schwarz inequality, we have

$$
\left(\sum_{x \in B_{u}(l)} g_{u}(x) d_{x}\right)^{2} \leqslant \operatorname{vol}\left(B_{u}(l)\right) \sum_{x \in B_{u}(l)} g_{u}^{2}(x) d_{x} .
$$

and therefore

$$
\alpha_{u}^{2} \leqslant \frac{\operatorname{vol}\left(B_{u}(l)\right)}{\operatorname{vol}(G)^{2}} \sum_{x} g_{u}^{2}(x) d_{x}
$$

By substitution into (28) and using (35), we have

$$
\begin{aligned}
\lambda_{1} \leqslant R\left(g_{u}^{\prime}\right) & \leqslant \frac{R(g)}{1-\frac{\operatorname{vol}\left(B_{u}(l)\right)}{\operatorname{vol}(G)}} \leqslant \frac{1-\sigma\left(1-\frac{1}{l+1}\right)}{1-\frac{\operatorname{vol}\left(B_{u}(l)\right)}{\operatorname{vol}(G)}} \\
& \leqslant 1-\sigma\left(1-\frac{1}{l+1}\right)+\frac{\operatorname{vol}\left(B_{u}(l)\right)}{\operatorname{vol}(G)} \\
& \leqslant 1-\sigma\left(1-\frac{c}{l+1}\right)
\end{aligned}
$$

The last inequality follows from Theorem 7 and the choice of $\epsilon=\sigma / k$. This completes the proof of Theorem 9 . 


\section{A lower bound for $\lambda_{n-1}$}

If a graph is bipartite, it is known (see [2]) that $\lambda_{i}=2-\lambda_{n-i-1}$ for all $0 \leqslant i \leqslant n-1$ and, in particular, $\lambda_{n-1}=2-\lambda_{0}=2$. If $G$ is not bipartite, it is easy to derive the following lower bound:

$$
\lambda_{n-1} \geqslant 1+1 /(n-1)
$$

by using the fact that the trace of $\mathcal{L}$ is $n$. This lower bound is sharp for the complete graph. However if $G$ is not the complete graph, is it possible to derive a better lower bound? The answer is affirmative. Here we give an improved lower bound for $\lambda_{n-1}$.

Theorem 10. In a connected graph $G=(V, E)$ with diameter $k$, the largest eigenvalue $\lambda_{n-1}$ of the normalized Laplacian $\mathcal{L}$ of $G$ satisfies

$$
\lambda_{n-1} \geqslant 1+\sigma\left(1-\frac{c}{k}\right)
$$

where $\sigma$ is as defined in (15), provided $k \geqslant c^{\prime} \log \sigma^{-1}$ and $\operatorname{vol}(G) \geqslant c^{\prime \prime} \sigma^{\log \sigma}$ for some absolute constants c's.

Proof. By definition, $\lambda_{n-1}$ satisfies

$$
\lambda_{n-1} \geqslant \frac{\sum_{x \sim y}(f(x)-f(y))^{2}}{\sum_{x} f^{2}(x) d_{x}}=R(f)
$$

for any $f: V \rightarrow \mathbb{R}$.

We will construct an appropriate $f$ such that $R(f) \geqslant 1+\sigma(1-c / \gamma)$ by considering the following function $f_{u}: V \rightarrow \mathbb{R}^{+}$, for a fixed vertex $u$, defined by

$$
\eta_{u}(x)= \begin{cases}(-1)^{t} \chi_{u}\left(\tilde{P}_{t}(x)\right)^{-1 / 2} & \text { if } \operatorname{dist}(u, x)=t \leqslant l \\ 0 & \text { otherwise }\end{cases}
$$

where $l \leqslant \gamma / 2$. Note that $\left|\eta_{u}(x)\right|=g_{u}(x)$ since we assume that $l \leqslant \gamma / 2$. Using the same proof in Claim A, we have

Claim A':

$$
\sum_{u} d_{u} \sum_{x} \eta_{u}^{2}(x) d_{x}=\sum_{j=0}^{l} \sum_{x} \sum_{\mathbf{p} \in \mathscr{P}_{u, x}^{(l)}} d_{u} w(\mathbf{p}) d_{x}=(l+1) \sum_{x} d_{x}^{2}
$$

Claim B':

$$
\sum_{u} d_{u} \sum_{x \sim y}\left(\eta_{u}(x)-\eta_{u}(y)\right)^{2} \geqslant(l+1+l \sigma) \sum_{x} d_{x}^{2}
$$


Proof of Claim B': The proof is quite similar to that of Claim B. For a fixed vertex $u$, the sum over unordered pair $\{x, y\}$ where $x \sim y$,

$$
\begin{aligned}
& \sum_{x \sim y}\left(\eta_{u}(x)-\eta_{u}(y)\right)^{2} \\
& \leqslant \sum_{t \leqslant l-1} \sum_{r \in V} \sum_{\substack{\mathbf{p} \in \mathscr{P}_{u, r}^{(t)} \\
\mathbf{p}^{\prime}=\mathbf{p} \cup s \in \mathscr{P}_{u, s}^{(t+1)}}}\left(\sqrt{w(\mathbf{p})}+\sqrt{w\left(\mathbf{p}^{\prime}\right)}\right)^{2}-\sum_{\mathbf{p} \in \mathscr{P}_{u, x}^{(l)}} w(\mathbf{p})\left(d_{x}-1\right) \\
& \leqslant \sum_{t \leqslant l-1} \sum_{x} \sum_{\mathbf{p} \in \mathscr{P}_{u, x}^{(t)}}\left(\sqrt{w(\mathbf{p})}+\sqrt{\frac{w(\mathbf{p})}{d_{x}-1}}\right)^{2}\left(d_{x}-1\right)-\sum_{\mathbf{p} \in \mathscr{P}_{u, x}^{(l)}} \sqrt{w(\mathbf{p})}\left(d_{x}-1\right) \\
& \leqslant \sum_{t \leqslant l-1} \sum_{x} \sum_{\mathbf{p} \in \mathscr{P}_{u, x}^{(t)}} w(\mathbf{p})\left(1+\frac{1}{d_{x}-1}+\frac{2}{\sqrt{d_{x}-1}}\right)\left(d_{x}-1\right)-\sum_{\mathbf{p} \in \mathscr{P}_{u, x}^{(l)}} w(\mathbf{p})\left(d_{x}-1\right) \\
& \leqslant \sum_{t \leqslant l-1} \sum_{x} \sum_{\mathbf{p} \in \mathscr{P}_{u, x}^{(t)}} w(\mathbf{p})\left(d_{x}+2 \sqrt{d_{x}-1}\right)-\sum_{\mathbf{p} \in \mathscr{P}_{u, x}^{(l)}} w(\mathbf{p})\left(d_{x}-1\right) .
\end{aligned}
$$

Using Fact 3, we have

$$
\begin{aligned}
& \sum_{u} d_{u} \sum_{x \sim y}\left(\eta_{u}(x)-\eta_{u}(y)\right)^{2} \\
& \geqslant \sum_{t \leqslant l-1} \sum_{u} d_{u} \sum_{\mathbf{p} \in \mathscr{P}_{u, x}^{(t)}} w(\mathbf{p})\left(d_{x}+2 \sqrt{d_{x}-1}\right)-\sum_{u} d_{u} \sum_{\mathbf{p} \in \mathscr{P}_{u, x}^{(l)}} w(\mathbf{p})\left(d_{x}-1\right) \\
& =l \sum_{x} d_{x}\left(d_{x}+2 \sqrt{d_{x}-1}\right)-\sum_{x} d_{x}^{2} \\
& =l(1+\sigma) \sum_{x} d_{x}^{2}-\sum_{x} d_{x}^{2} \\
& =(l-1+l \sigma) \sum_{x} d_{x}^{2}
\end{aligned}
$$

This proves Claim B'.

Combining Claims A' and B', we have

$$
\begin{aligned}
& \sum_{u} d_{u} \sum_{x \sim y}\left(\eta_{u}(x)-\eta_{u}(y)\right)^{2} \\
& \geqslant(l-1+l \sigma) \sum_{x} d_{x}^{2} \\
& \geqslant(l-1+l \sigma)\left(\frac{1}{l+1}\right) \sum_{u} d_{u} \sum_{x} \eta_{u}^{2}(x) d_{x} \\
& =\left(1+\frac{l \sigma}{l-1}\right) \sum_{u} d_{u} \sum_{x} \eta_{u}^{2}(x) d_{x}
\end{aligned}
$$


Thus we deduce that there is a vertex $u$ such that

$$
\begin{aligned}
R\left(\eta_{u}\right) & =\frac{\sum_{x \sim y}\left(\eta_{u}(x)-\eta_{u}(y)\right)^{2}}{\sum_{x} \eta_{u}^{2}(x) d_{x}} \\
& \leqslant 1+\frac{l \sigma}{l-1} .
\end{aligned}
$$

We consider the function $\eta_{u}^{\prime}$ defined by

$$
\eta_{u}^{\prime}(x)=\eta_{u}(x)-\alpha_{u}
$$

where

$$
\alpha_{v}=\frac{\sum_{x} \eta_{v}(x) d_{x}}{\sum_{x} d_{x}}=\frac{\sum_{x} \eta_{v}(x) d_{x}}{\operatorname{vol}(G)}
$$

so that $\eta_{u}^{\prime}$ satisfies the condition that

$$
\sum_{x} \eta_{u}^{\prime}(x) d_{x}=0
$$

Hence, we have

$$
\begin{aligned}
\lambda_{n-1} \geqslant R\left(\eta_{u}^{\prime}\right) & =\frac{\sum_{x \sim y}\left(\eta_{u}^{\prime}(x)-\eta_{u}^{\prime}(y)\right)^{2}}{\sum_{x} \eta_{u}^{\prime 2}(x) d_{x}} \\
& =\frac{\sum_{x \sim y}\left(\eta_{u}(x)-\eta_{u}(y)\right)^{2}}{\sum_{x} \eta_{u}^{2}(x) d_{x}-\alpha_{u}^{2} \operatorname{vol}(G)} \\
& \geqslant 1+\sigma\left(1+\frac{c}{l}\right)-\frac{\operatorname{vol}\left(B_{u}(l)\right)}{\operatorname{vol}(G)} .
\end{aligned}
$$

This completes the proof of Theorem 10 .

\section{References}

[1] M. Capalbo, O. Reingold, S. Vadhan and A. Wigderson. Randomness conductors and constant-degree lossless expanders, Proceedings of the 34th Annual ACM symposium on Theory of Computing, 659-668, 2002.

[2] F. Chung, Spectral Graph Theory, AMS Publications, vii+207 pages, 1997.

[3] J. Friedman, A proof of Alon's second eigenvalue conjecture and related problems, Mem. Amer. Math. Soc., 195, viii+100 pages, 2008.

[4] N. Kahale, Eigenvalues and expansion of regular graphs, JACM, 42 (5): 1091-1106, 1995.

[5] P. Hall, On representatives of subsets, J. London Math. Soc. 10 (1): 26-30, 1935. 
[6] S. Hoory, A lower bound on the spectral radius of the universal cover of a graph, $J$. Combin. Theory B, 93: 33-43, 2005.

[7] S. Hoory, N. Linial and A. Wigderson, Expander graphs and their applications, Bull. Amer. Math. Soc., 43: 439-561, 2006.

[8] A. Nilli, On the second eigenvalue of a graph, Discrete Math., 91: 207-210, 1991.

[9] R. M. Tanner, Explicit construction of concentrators from generalized $n$-gons, SIAM J. Algebraic Discrete Methods, 5: 287-294, 1984.

[10] S. J. Young, The weighted spectrum of the universal cover and an Alon-Boppana result for the normalized Laplacian, preprint. 


\section{Corrigendum - added 3th November 2017}

1. In the abstract, line $6-8$, the statement of the main result should be replaced by

$$
\lambda_{1} \leqslant 1-\sigma\left(1-\frac{5}{k}\right)
$$

provided $\sigma=2 \sum_{v} d_{v} \sqrt{d_{v}-1} / \sum_{v} d_{v}^{2} \leqslant 1 / 2$ and $k(1.5)^{k} \geqslant \sigma^{-1}$ where $d_{v}$ denotes the degree of the vertex $v$ with minimum degree at least 2 .

Also, page 12, line -3 to -1 , the statement of Theorem 9 should be similarly replaced as above.

2. Page 3 , line 13 , the constant $c$ should be replaced by 5 .

3. Page 9, line -9. "... for some constant c." should be replaced by "... for $c=$ $1 / \log 1.5 . "$

4. Page 9, line -6 . Replace "... largest ..." by "... least ...".

5. Page 10 , line $3, \bar{s}_{j}$ should be replaced by $\bar{s}_{j+1}$.

6. Page 3, line 7 to 11. Delete "We set ... as defined in (15)." Note that $\epsilon$ was defined later near the end of the proof of Theorem 9.

7. Page 16, line -6 , replace “... using $(35), \ldots$ ” by “... using $(27), \ldots$ ”.

8. Page 16, line -3. Replace " $c /(l+1)$ " by " $5 / k$ ".

9. Page 16, line -2. Replace " ... the choice of $\epsilon=\sigma / k$." by " ... the choice of $\epsilon=\sigma / k$ which satisfies $k \geqslant\left(\log \epsilon^{-1}\right) / \log 1.5$."

10. Page 17, line 11 to line 13, the statement of Theorem 10 should be replaced by

$$
\lambda_{n-1} \geqslant 1+\sigma\left(1-\frac{5}{k}\right)
$$

provided $\sigma=2 \sum_{v} d_{v} \sqrt{d_{v}-1} / \sum_{v} d_{v}^{2} \leqslant 1 / 2$ and $k(1.5)^{k} \geqslant \sigma^{-1}$ where $d_{v}$ denotes the degree of the vertex $v$ with minimum degree at least 2 . 